\title{
Inverse-chirp signals and spontaneous scalarisation with self-interacting potentials in stellar collapse
}

\author{
Roxana Rosca-Mead ${ }^{1}$, Christopher J Moore ${ }^{2}$, Michalis \\ Agathos $^{3}$, Ulrich Sperhake ${ }^{1,4}$ \\ ${ }^{1}$ Department of Applied Mathematics and Theoretical Physics, Centre for \\ Mathematical Sciences, University of Cambridge, Wilberforce Road, Cambridge \\ CB3 0WA, United Kingdom \\ ${ }^{2}$ Institute for Gravitational Wave Astronomy and School of Physics and \\ Astronomy, University of Birmingham, Edgbaston, Birmingham B15 2TT, UK \\ 3 Theoretical Physics Institute, University of Jena, 07743 Jena, Germany \\ ${ }^{4}$ Theoretical Astrophysics 350-17, California Institute of Technology, $1200 \mathrm{E}$ \\ California Boulevard, Pasadena, CA 91125, USA \\ E-mail: rr417@cam.ac.uk
}

\begin{abstract}
We study how the gravitational wave signal from stellar collapse in scalar-tensor gravity varies under the influence of scalar self-interaction. To this end, we extract the gravitational radiation from numerical simulations of stellar collapse for a range of potentials with higher-order terms in addition to the quadratic mass term. Our study includes collapse to neutron stars and black holes and we find the strong inverse-chirp signals obtained for the purely quadratic potential to be exceptionally robust under changes in the potential at higher orders; quartic and sextic terms in the potential lead to noticeable differences in the wave signal only if their contribution is amplified, implying a relative fine-tuning to within 5 or more orders of magnitude between the mass and self-interaction parameters.
\end{abstract}




\section{Introduction}

In spite of the tremendous success of general relativity (GR) in explaining a plethora of phenomena in the observable universe [1,2], theoretical considerations as well as persistent puzzles in observational astronomy may ultimately require Einstein's theory to be extended or modified [3]. The new era of gravitational wave (GW) astronomy, marked by LIGO's detection of GW150914 [4] and ten further events identified since [5], in the data of the LIGO [6] and Virgo [7] detectors, has now opened up qualitatively new opportunities to probe GR in the strong-field regime [8]. First tests using GW observations have resulted in valuable constraints on deviations from GR, including constraints on the post-Newtonian coefficients and on the propagation of the dipole radiation from compact binaries [9, 10,11], but more comprehensive tests will require more systematic efforts on the modelling of sources of GWs in modified theories of gravity.

This effort faces considerable challenges. First, there should exist a well-posed initial value formulation of the theory under consideration to ensure that solutions are unique and depend continuously on the initial data. This aspect has been explored for a few candidate theories $[12,13,14,15]$ but largely remains an open issue which is further complicated by the fact that the question of well-posedness is, in general, gauge dependent. The difficulties associated with the potential lack of well-posedness may be bypassed through a perturbative expansion of the theory around GR and truncating this series at some order in the expansion parameter. Such an effective-field theory approach has been used to compute deviations at linear order from GR in the inspiral of black-hole (BH) binaries in dynamical Chern-Simons theory [16] and scalar GaussBonnet gravity [17]. A second major challenge faced in the modelling of sources of GWs in modified gravity arises from the tremendous success of GR itself. Clearly, a serious candidate theory must be compatible with the wide range of observational tests GR has already passed. The challenge then is to identify theories that agree with GR in the weak-field regime but make concrete predictions in the strong-field regime that deviate sufficiently from GR such that they serve as a potential discriminant in observational tests [3].

The most concrete prediction of this type is the spontaneous scalarisation of compact stars in scalar tensor (ST) theory identified by Damour and Esposito-Farèse [18]. Here, a second branch of strongly scalarised stars emerges over a significant subset of the parameter space of the theory and its members may be energetically favoured over their weakly scalarised, "GR like" counterparts. The onset of this strong scalarisation has been observed in various simulations of collapsing stars and binary neutron star mergers in ST theory where it generates significant gravitational radiation in the form of scalar waves [19, 20, 21, 22, 23, 24, 25, 26]. All of these studies, however, as well as recent analytical calculations of binary dynamics in ST theory to high post-Newtonian order [27, 28], are restricted to the case of massless ST gravity. In the massless case, the parameter space of the theory is already severely constrained through binary pulsar observations [29, 30,31] and Doppler tracking of the Cassini spacecraft [32]. The surviving parameter regime of massless ST gravity barely allows for spontaneous scalarisation to occur.

On the other hand, both the binary pulsar and Cassini constraints rely on observations of widely separated objects and therefore do not apply to theories where the scalar degree of freedom is effectively screened on the scales in question. Such a screening is provided by a scalar mass $\mu \gtrsim 10^{-19} \mathrm{eV}$ corresponding to a Compton 
wavelength $\lambda_{c}=(2 \pi \hbar) /(\mu c)$ smaller than or comparable to the distances between the relevant objects [33, 34].

Massive ST gravity therefore remains a largely untested class of theories with considerable potential to generate strong-field deviations from GR in, as yet, unobserved regimes while passing all weak-field tests. The theory is furthermore manifestly well-posed by virtue of the equivalence of its description in the Einstein and Jordan frames $[35,36,37,38]$. Recent years have accordingly seen a rising number of studies exploring potentially observable features of compact objects in this class of theories, such as the computation of static equilibrium models [34], the structure of uniformly or differentially rotating neutron stars and their inertia and quadrupole moment $[39,40,41]$ and the generalisation of the spontaneous scalarisation mechanism to a wider range of theories [42, 43, 44].

In previous work [45], we have computed the first GW signals in massive ST gravity and demonstrated how the gravitational collapse of stellar cores leads to highly characteristic signals, stretched out over years or even centuries by the dispersive nature of the mass term, that would show up in existing LIGO-Virgo searches. Most recently, a first exploration of stellar collapse in ST theory with a mass and a quartic self-interaction term has identified the possibility of a weakening effect of the selfinteraction term on the magnitude of the GW signal [46]. The main purpose of this study is to perform a systematic exploration of the impact of higher-order terms in the scalar potential and test the robustness of the inverse-chirp signals found in [45]. As we will demonstrate in the remainder of this paper, the generation of the signals, their amplitude and propagation is astonishingly robust to modifications of the potential.

This study is organised as follows. In Sec. 2 we review the formalism of our simulations, with a focus on the modifications in the scalar potential. The generation of GW signals in stellar collapse is modelled and compared to the case of noninteracting scalars in Sec. 3. In Sec. 4, we discuss the propagation of the signal from radii comparable to our computational domain to large distances in the far-field region. Throughout this work, we use natural units where the speed of light and gravitational constant $c=G=1$.

\section{Formalism}

We consider in this work the class of ST theories of gravity first studied by Bergmann [47] and Wagoner [48] which are characterised by the following properties. The theory is described by an action $S=S_{\mathrm{G}}+S_{\mathrm{M}}$, where $S_{\mathrm{G}}$ only contains the gravitational fields and $S_{\mathrm{M}}$ describes the matter fields and their interaction with gravity. Gravity is mediated by the (physical) spacetime metric $g_{\alpha \beta}$ and a single, non-minimally coupled real scalar field. Variation of the action $S$ results in at most two-derivative field equationsł that are diffeomorphism invariant and obey the weak equivalence principle. This class of theories is conveniently described in the so-called Einstein frame, obtained from the physical or Jordan frame through a conformal transformation of the metric and a redefinition of the scalar degree of freedom. In natural units, the general action

¥ The word "two-derivative" implies that each term may involve at most two derivative operators, i.e. terms may be linear in second derivatives or quadratic in first derivatives but may not contain products such as $f_{, \alpha} f, \beta \gamma$. 
for these theories is given by $[49,3]$

$$
S=\int \mathrm{d} x^{4} \frac{\sqrt{-\bar{g}}}{16}\left[\bar{R}-2 \bar{g}^{\alpha \beta} \partial_{\alpha} \varphi \partial_{\beta} \varphi-4 V(\varphi)\right]+S_{\mathrm{M}}\left(g_{\alpha \beta}, \psi_{\mathrm{M}}\right),
$$

where $\varphi$ is the scalar field, $\psi_{\mathrm{M}}$ collectively denotes all matter fields, and $\bar{R}$ and $\bar{g}$ are the Ricci scalar and metric determinant constructed from the conformal metric

$$
\bar{g}_{\alpha \beta}=F(\varphi) g_{\alpha \beta} .
$$

In the Einstein frame, the scalar field is minimally coupled to the conformal metric $\bar{g}_{\alpha \beta}$ but the matter fields couple to the physical or Jordan-Fierz metric $g_{\alpha \beta}$. This class of theories has two free functions, the scalar potential $V(\varphi)$ and the conformal factor $F(\varphi)$; we will discuss both in more detail below.

Our study concerns the gravitational collapse of stellar cores at the end of their nuclear burning phase and the GW signal generated in the rapid transition of a lowdensity star to a high-density compact object. The main dynamics of this process are dominated by the sudden radial compression of the stellar matter and we therefore employ spherical symmetry in this study. More specifically, we use radial gauge and polar slicing in the Einstein frame and write the conformal metric as

$$
\bar{g}_{\alpha \beta} \mathrm{d} x^{\alpha} \mathrm{d} x^{\beta}=-F \alpha^{2} \mathrm{~d} t^{2}+F X^{2} \mathrm{~d} r^{2}+r^{2}\left(\mathrm{~d} \theta^{2}+\sin ^{2} \theta \mathrm{d} \phi^{2}\right),
$$

where $\alpha$ and $X$ are functions of $(t, r)$. We model the stellar matter as a perfect fluid with baryon density $\rho$, pressure $P$, internal energy $\epsilon$, and 4-velocity $u^{\alpha}=$ $\left(1-v^{2}\right)^{-1 / 2}\left[\alpha^{-1}, v X^{-1}, 0,0\right]$. Here, $\rho, P, \epsilon$ and $v$ are also functions of $(t, r)$, and the energy momentum tensor is given by

$$
T_{\alpha \beta}=(\rho+\rho \epsilon+P) u_{\alpha} u_{\beta}+P g_{\alpha \beta} .
$$

Following Refs. [26, 45], we use a hybrid equation of state (EOS) where the pressure consists of a cold and a thermal part, $P=P_{\mathrm{c}}+P_{\mathrm{th}}$, defined in terms of the cold contribution to the internal energy $\epsilon_{c}$ by

$$
\begin{array}{ll}
\rho \leq \rho_{\text {nuc }}: & P_{\mathrm{c}}=K_{1} \rho^{\Gamma_{1}}, \quad \epsilon_{\mathrm{c}}=\frac{K_{1}}{\Gamma_{1}-1} \rho^{\Gamma_{1}-1}, \\
\rho>\rho_{\text {nuc }}: & P_{\mathrm{c}}=K_{2} \rho^{\Gamma_{2}}, \quad \epsilon_{\mathrm{c}}=\frac{K_{2}}{\Gamma_{2}-1} \rho^{\Gamma_{2}-1}+E_{3}, \\
P_{\mathrm{th}}=\left(\Gamma_{\mathrm{th}}-1\right) \rho\left(\epsilon-\epsilon_{\mathrm{c}}\right) . &
\end{array}
$$

Here we use a nuclear density $\rho_{\text {nuc }}=2 \times 10^{14} \mathrm{~g} \mathrm{~cm}^{-3}, K_{1}=4.9345 \times 10^{14}[\mathrm{cgs}]$ [50], and $K_{2}$ and $E_{3}$ follow from continuity across $\rho_{\text {nuc }}$. There thus remain 3 free parameters determining the EOS, the polytropic exponents $\Gamma_{1}, \Gamma_{2}$ and the thermal coefficient $\Gamma_{\text {th }}$. In the remainder of this work we set these to the fiducial values $\Gamma_{1}=1.3$, $\Gamma_{2}=2.5, \Gamma_{\mathrm{th}}=1.35$ of Ref. [26]; see Sec. 3.1 and references therein for further details. By performing additional simulations, we have found that the impact of the self-interaction terms is not significantly affected by varying the EOS.

The field and matter equations determining the time evolution of an initial stellar profile with the above EOS in ST theory and spherical symmetry are then given by 
Eqs. (3)-(6) in Ref. [45]. The key difference to that study is that we now consider a wider class of scalar potentials,

$$
V(\varphi)=\frac{\mu^{2} \varphi^{2}}{2 \hbar^{2}}\left(1+\lambda_{1} \frac{\varphi^{2}}{2}+\lambda_{2} \frac{\varphi^{4}}{3}+\ldots+\lambda_{n} \frac{\varphi^{2 n}}{n+1}\right), \quad \text { with } \quad \lambda_{n}>0 .
$$

Here, the scalar mass $\mu$ introduces a characteristic frequency

$$
\omega_{*}=2 \pi f_{*}=\frac{\mu}{\hbar} .
$$

In this work we set $\mu=10^{-14} \mathrm{eV}$ corresponding to $\omega_{*}=15.2 \mathrm{~s}^{-1}$ or $f_{*}=2.42 \mathrm{~Hz}$. Note that all $\lambda_{i}$ in Eq. (7) are dimensionless and that we recover the massive but not self-interacting case of [45] by setting $\lambda_{i}=0$, and the massless case by setting $\mu=0$.

The remaining free function in our theory is the conformal factor or coupling function $F(\varphi)$. The standard choice to parametrize this function, $\S$

$$
F(\varphi)=e^{-2 \alpha_{0} \varphi-\beta_{0} \varphi^{2}}
$$

is motivated by the fact that in this form, $\alpha_{0}$ and $\beta_{0}$ completely determine all modifications of gravity at first post-Newtonian order $[35,36,51]$. In the remainder of our study we will follow this choice and work with the conformal factor in Eq. (9).

The parameter $\alpha_{0}$ is related to the logarithmic derivative of the conformal factor,

$$
\alpha_{0}=-\left.\frac{1}{2} \frac{\mathrm{d} \ln F}{\mathrm{~d} \varphi}\right|_{\varphi=0}
$$

and determines the coupling of the scalar field to a GW detector far away from the source: A scalar wave of angular frequency $\Omega$ induces a total detector strain

$$
h=2 \alpha_{0}\left[1-\left(\frac{\omega_{*}}{\Omega}\right)\right] \varphi .
$$

For massless fields, this reduces to the familiar $h=2 \alpha_{0} \varphi$, but for a massive scalar, the strain is mildly reduced because the scalar mode splits into a transverse and a longitudinal component; see [45] for more details.

Let us conclude this section with a summary of the free parameters in our ST theory. The conformal factor is described by $\alpha_{0}$ and $\beta_{0}$ and we have $n$ further parameters describing the self-interaction of the scalar field. In this paper we restrict our attention to the case $n=2$ giving a total of four free parameters, $\alpha_{0}, \beta_{0}, \lambda_{1}, \lambda_{2}$, additionally to the four fixed parameters $\mu=10^{-14} \mathrm{eV}, \Gamma_{1}=1.3, \Gamma_{2}=2.5, \Gamma_{\mathrm{th}}=1.35$ for the scalar mass and the EOS.

\section{Models and results}

The computational framework for our core collapse simulations is based on the open source code GR1D [52] for modelling spherically symmetric fluids in GR with highresolution shock capturing schemes. In Refs. [26] and [45], GR1D has been extended to ST gravity with a massless or a massive scalar field, respectively. Here we employ the same setup as used in [45] except that the scalar potential is now given by Eq. (7). The runs presented in this paper have been performed with an inner uniform grid of

$\S$ A common alternative notation for the conformal factor is $A(\varphi):=F(\varphi)^{-1 / 2}$. 

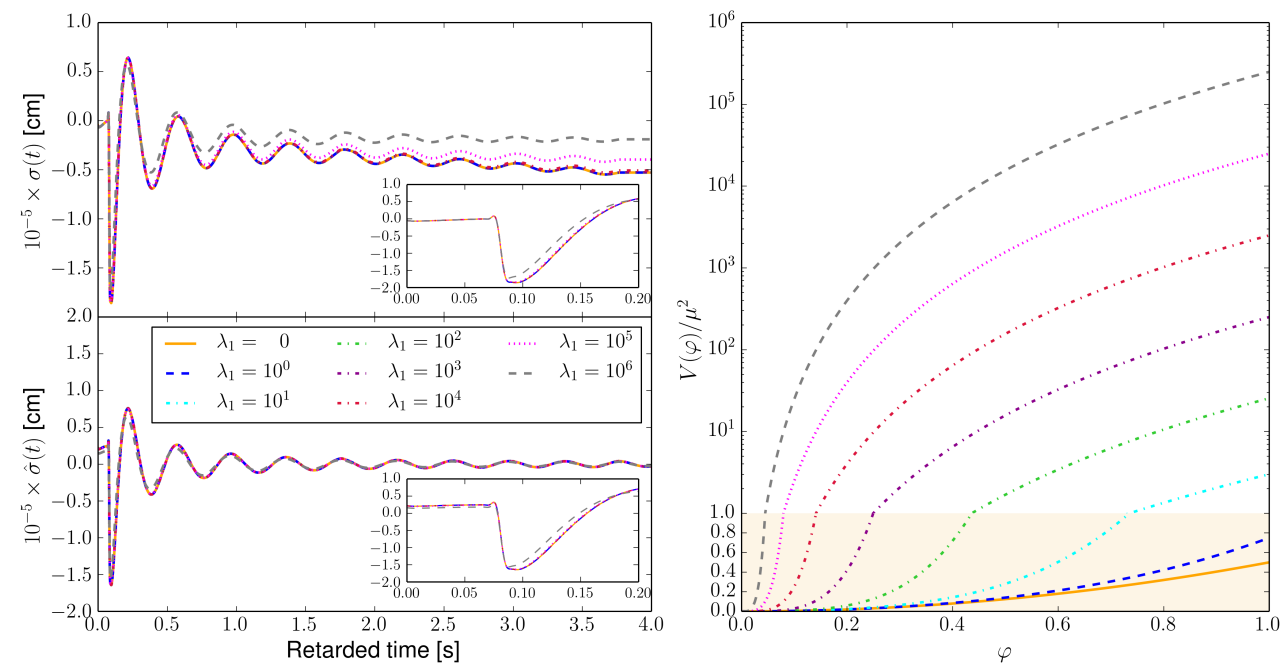

Figure 1. The effect of varying the quartic, $\lambda_{1}$, term of the scalar field potential. Top-left panel: the scalar field signal at the extraction radius $7 \times 10^{4} \mathrm{~km}$. Bottomleft panel: the band-passed scalar field signal at the extraction radius $7 \times 10^{4} \mathrm{~km}$; modes with frequencies less than $\omega_{*}$ are set to 0. Right-hand panel: the scalar field potential. The progenitor used is a $41 M_{\odot}$ star of $10^{-4}$ solar metallicity and the scalar parameters are $\alpha_{0}=10^{-2}, \beta_{0}=-20$.

$\Delta r=250 \mathrm{~m}$ up to $r=40 \mathrm{~km}$ and an outer logarithmic grid up to $r=9 \times 10^{5} \mathrm{~km}$ with a total of 10000 grid points. At this resolution, the waveforms incur a discretization error of about $6 \%$ [45].

The initial data for our simulations are given by the catalogue of non-rotating pre-super-nova stellar profiles of Woosley and Heger [53, 54] with the scalar field and its time derivative initialised to zero. This catalogue covers progenitor models with zero-age-main-sequence (ZAMS) masses ranging between $(12-75) M_{\odot}$ and different metallicity. Here we focus on two stellar models with $10^{-4}$ times solar metalicity and $M_{\text {ZAMS }}=39 M_{\odot}$ and $41 M_{\odot}$. We have studied a wider range of initial models and also tested the effect of varying the EOS parameters; the impact of the self-interaction terms $\lambda_{i}$ on the GW generation exhibits a universal character in all these simulations which is fully encapsulated by the models presented in this section.

The one common feature of all our simulations is that they result in strong scalarisation of the compact stars formed during the collapse and, thus, lead to a large GW signal for ST theory without self-interaction, i.e. for $\lambda_{i}=0$. Let us first consider the collapse of a $41 M_{\odot}$ progenitor model with $10^{-4}$ solar metallicity with ST parameters $\alpha_{0}=10^{-2}, \beta_{0}=-20$. For $\lambda_{i}=0$, the collapse of the baryon matter leads to core bounce at $t \simeq 0.07 \mathrm{~s}$ which generates a scalar wave signal $\sigma=r_{\mathrm{ex}} \varphi$ of $O\left(10^{5}\right)$ at extraction radius $r_{\mathrm{ex}}=7 \times 10^{4} \mathrm{~km}$. This signal is shown as the solid (orange) curve in Fig. 1. During the collapse, the scalar field at the centre of the star rapidly increases before levelling off at a magnitude of 0.38 .

Next, we repeat this collapse simulation for non-zero $\lambda_{1}$ but keeping all $\lambda_{i}=0$ for $i \geq 2$. Even though the potential changes dramatically as we increase $\lambda_{1}$ (see the right panel of Fig. 1), up to $\lambda_{1}=10^{4}$, we observe no modifications in the scalar wave signals 

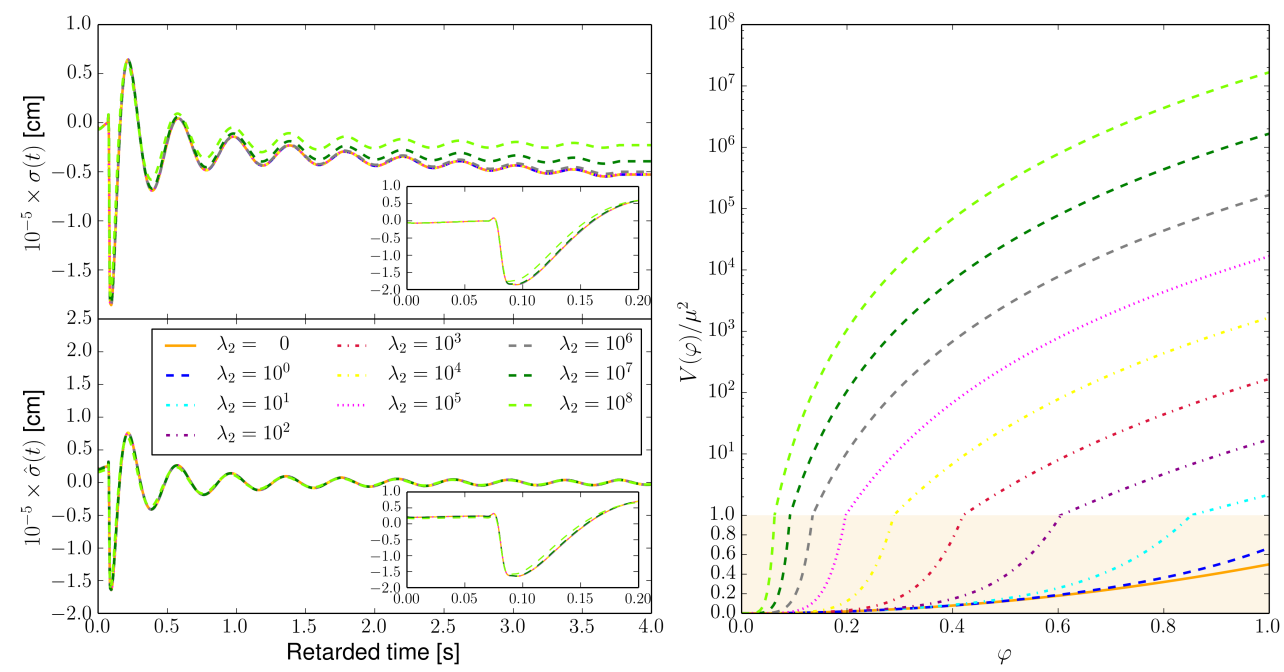

Figure 2. The effect of varying the sextic, $\lambda_{2}$, term of the scalar field potential. Top-left panel: the scalar field signal at the extraction radius $7 \times 10^{4} \mathrm{~km}$. Bottomleft panel: the band-passed scalar field signal at the extraction radius $7 \times 10^{4} \mathrm{~km}$; modes with frequencies less than $\omega_{*}$ are set to 0. Right-hand panel: the scalar field potential. The progenitor used is a $41 M_{\odot}$ star of $10^{-4}$ solar metallicity and the scalar parameters are $\alpha_{0}=10^{-2}, \beta_{0}=-20$.

(see upper left panel of Fig. 1) and the peak value of the scalar field at the stellar centre remains 0.38 . For $\lambda_{1} \gtrsim 10^{5}$, we start seeing a mild deviation of the signal in the form of a slow drift. The low frequency modes associated with this drift, however, will be screened during the propagation of the signals to large distances: all modes with frequencies below $\omega_{*}-2.42 \mathrm{~Hz}$ in this case - decay exponentially at large radii [45]. In the context of LIGO-Virgo observations, these modes are therefore irrelevant. In order to asses the observationally relevant impact of $\lambda_{1}$, we band pass the signals by suppressing modes below $\omega_{*}$. The result is shown in the bottom left panel of Fig. 1 and demonstrates excellent agreement of all waveforms up to $\lambda_{1} \approx 10^{6}$. Increasing $\lambda_{1}$ beyond this magnitude leads to a gradual reduction in the amplitude of the scalar radiation.

In Fig. 2, we have repeated the same analysis but now varying $\lambda_{2}$ while keeping all other $\lambda_{i}=0$. The observations are the same as for $\lambda_{1}$ except that the sextic term only exhibits visible deviations for $\lambda_{2} \gtrsim 10^{7}$ (instead of $\lambda_{1} \gtrsim 10^{5}$ for the quartic term). This quantitative difference is not surprising given the additional suppression in the sextic term by a factor $\sim \varphi^{2}$. We have obtained similar results when varying $\lambda_{3}$ : we observe no significant deviation up to values of $\lambda_{3} \approx 10^{8}$ but a reduction of the GW amplitudes above this value. For $\lambda_{3}=10^{12}$, for example, we see a drop by about $50 \%$ relative to the non-self-interacting case.

The scenario discussed so far represents a straightforward collapse of a lowdensity star to a compact neutron star. Especially for high $M_{\text {zAms }}$ progenitor models, however, the outcome can reveal a more complex behaviour. The collapse may first form a weakly scalarised neutron star which, through continued accretion, later migrates to the strongly scalarised branch or the collapse may form a $\mathrm{BH}$ instead of a 

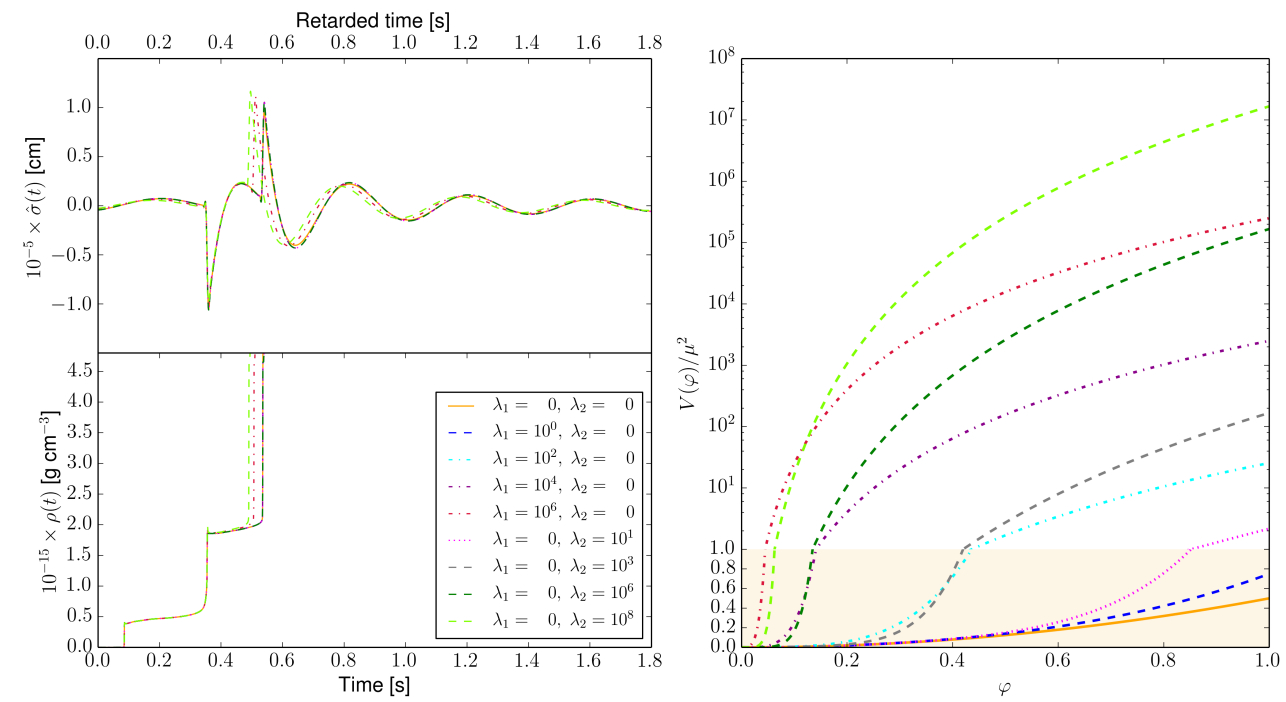

Figure 3. The effect of varying the $\lambda_{1}$ and $\lambda_{2}$ parameters for models that lead to BH formation. Top-left panel: the band-passed scalar field signal at the extraction radius $7 \times 10^{4} \mathrm{~km}$; modes with frequencies less than $\omega_{*}$ are set to 0 . Bottom-left panel: the central density until a $\mathrm{BH}$ is reached. Right-hand panel: the scalar field potential. The progenitor used is a $39 M_{\odot}$ star of $10^{-4}$ solar metallicity and the scalar parameters are $\alpha_{0}=10^{-3}, \beta_{0}=-5$.

neutron star. We now consider a model that exhibits all these features in one collapse. This model consists of a $39 M_{\odot}$ progenitor with $10^{-4}$ solar metallicity collapsing in ST theory with $\alpha_{0}=10^{-3}, \beta_{0}=-5$. For $\lambda_{i}=0$, this star initially forms a weakly scalarised compact core, as indicated by the first jump in the central density at $t \simeq 0.09 \mathrm{~s}$ in the bottom left panel of Fig. 3 . Through accretion, the core becomes more massive and eventually migrates to the strongly scalarised branch at $t \simeq 0.35 \mathrm{~s}$. This change in scalarisation - the central value of the scalar field increases from nearly zero to 0.24 - generates the first peak in the scalar wave signal in the upper left panel of Fig. 3. As the core continues accreting and becomes yet more massive, ultimately a $\mathrm{BH}$ is formed and the star descalarises in accordance with the no-hair theorems for BHs $[55,56]$. From the viewpoint of GW generation, this process is, in essence, a reversal of the original scalarisation and, correspondingly, leads to a second peak at $t \simeq 0.53 \mathrm{~s}$ resembling a mirror image of the first peak. This scenario remains largely unchanged when increasing $\lambda_{1}$ up to $10^{5}$ or, alternatively, $\lambda_{2}$ up to $10^{7}$. For larger values, we eventually see a shift in the time of collapse to a BH: $t \simeq 0.50 \mathrm{~s}$ for $\lambda_{1}=10^{6}$ and at $t \simeq 0.49 \mathrm{~s}$ for $\lambda_{2}=10^{8}$. For even larger values of the quartic and sextic parameters, the scalarisation is weakened in accordance with our observations in the above cases. Furthermore, the strongly scalarised stage becomes shorter; for $\lambda_{1}=10^{7}$ or $\lambda_{2}=10^{9}$ this stage disappears and the star collapses directly into a $\mathrm{BH}$ at $t \simeq 0.35 \mathrm{~s}$. This observation indicates that strong scalarisation tends to delay $\mathrm{BH}$ formation; we will discuss this phenomenon in more detail in a follow-up paper [57]. 


\section{Self-interaction and wave propagation}

The GW signal we may observe from a stellar collapse event in massive ST gravity is affected by self-interaction terms in the potential in two ways: (i) the scalarisation of the star and the corresponding local generation of a GW signal, and (ii) the propagation of this signal from source to detector. In the previous section, we have addressed item (i); GW generation is affected by the self-interaction terms only for enormous values of the dimensionless coefficients $\lambda_{1}$ and $\lambda_{2}$. In this section we will discuss item (ii). We begin by reviewing the case without self-interaction.

In ST theories with $\lambda_{i}=0$, the GW signals at astrophysically large distances, $d$, tend to a predictable inverse chirp [45, 57]; at each instant in time the signal is quasi-monochromatic with instantaneous frequency

$$
\Omega(t)=\omega_{*} / \sqrt{1-(d / t)^{2}} \text { for } t>d,
$$

and an amplitude which varies as a function of time according to

$$
A(t)=\sqrt{\frac{2}{\pi}} \frac{\left(\Omega^{2}-\omega_{*}^{2}\right)^{3 / 4}}{\omega_{*} d^{1 / 2}}|\tilde{\sigma}[\Omega(t)]| .
$$

Here, $\sigma(t) \equiv r_{\mathrm{ex}} \varphi\left(t ; r_{\mathrm{ex}}\right)$ is the rescaled scalar field signal extracted at a radius $r_{\mathrm{ex}}$ in the wave zone of a strong-field simulation (e.g. as obtained from our numerical simulations at $7 \times 10^{4} \mathrm{~km}$ ), and a tilde denotes a Fourier transform. Note that the scalar profile near the source only enters into the expression for the amplitude of the signal at large distances (not the frequency), and even then only through the modulus of its Fourier transform (or power spectrum).

\subsection{A toy model}

The structure of the inverse chirp is due, almost entirely, to the dispersive nature of the wave propagation, and not to any internal dynamics of the neutron star. To emphasise this point let us consider the following toy model.

At large distances, the dynamics of the non-self-interacting scalar field are governed by the flat-space Klein-Gordon equation,

$$
\left(\partial_{t}^{2}-\nabla^{2}\right) \varphi+\mu^{2} \varphi=4 \pi \varrho,
$$

where $\varrho$ is the source for the scalar field. Although this flat-space equation is only expected to hold at large radii, in our toy model it will be used throughout spacetime. Before the neutron star has scalarised there is no source term; however, after scalarisation the field is sourced by a small, static neutron star. We approximate this scenario with a source function given by

$$
\varrho(t ; \boldsymbol{x})=\varphi_{*} W_{\tau}(t) \delta^{(3)}(\boldsymbol{x}), \quad \text { where } W_{\tau}(t)=\left\{\begin{array}{ll}
\frac{2}{\pi} \arctan \left(\frac{t}{\tau}\right) & \text { if } t \geq 0 \\
0 & \text { if } t<0
\end{array},\right.
$$

where $\varphi_{*}(x)$ parameterises the magnitude of the scalarisation, and $\tau$ is the typical timescale over which scalarisation occurs. The field equation for our toy model, Eq. (14), is linear and may be solved using a retarded Green's function;

$$
\varphi(x)=\int \mathrm{d}^{4} x G\left(x, x^{\prime}\right) \varrho\left(x^{\prime}\right),
$$


where $G\left(x, x^{\prime}\right)$ is given explicitly by [58]

$$
G\left(x, x^{\prime}\right)=\left[\delta(\chi)-\frac{\mu}{\sqrt{-2 \chi}} J_{1}(\mu \sqrt{-2 \chi}) \theta(-\chi)\right] \quad \text { if } t-t^{\prime} \geq 0 \quad \text { else } 0,
$$

with $\chi\left(x, x^{\prime}\right)=\frac{1}{2} \eta_{\mu \nu}\left(x-x^{\prime}\right)^{\mu}\left(x-x^{\prime}\right)^{\nu}$ and $J_{\alpha}$ denoting the Bessel functions of the first kind. Evaluating the integral in Eq. (16) with the source in Eq. (15) gives

$$
\varphi(t ; r)=\varphi_{*}\left[\frac{W_{\tau}(t-r)}{r}-\int_{0}^{t-r} \mathrm{~d} t^{\prime} \frac{\mu J_{1}\left(\mu \sqrt{\left(t-t^{\prime}\right)^{2}-r^{2}}\right) W_{\tau}\left(t^{\prime}\right)}{\sqrt{\left(t-t^{\prime}\right)^{2}-r^{2}}}\right] \quad \text { if } t \geq r \quad \text { else } 0 .
$$

This is the solution (written in terms of an integral to be evaluated numerically) for the scalar field in our toy model; the first term is the familiar result for a massless field, whilst the second "tail" term depends on $\mu$ and accounts for the dispersive nature of the wave propagation. The result in Eq. (18) is plotted as a function of retarded time

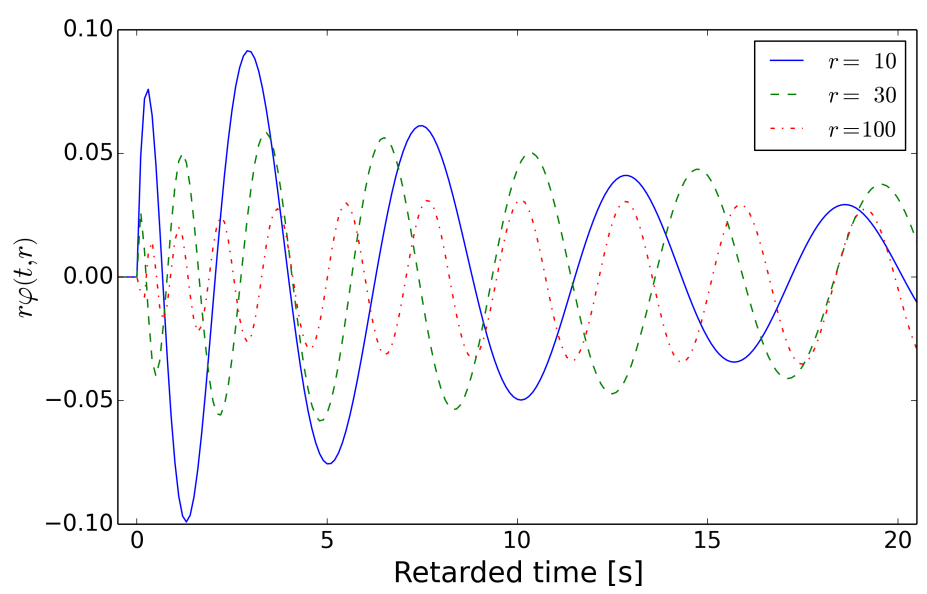

Figure 4. The scalar field as a function of retarded time in the toy model [Eq. (18)] at three different distances from the origin: $r=10,30$, and 100 . The other model parameter values are $\mu=\varphi_{*}=\tau=1$, and $\lambda_{i}=0$. As described in [45], the signal becomes increasingly oscillatory at greater distances; the $r=10$ curve has five maxima in the plot range, the $r=30$ curve has seven, whilst the $r=100$ curve has ten.

$t-r$ at several fixed radii in Fig. 4. Obviously our simple toy model has neglected all of the interesting physics in the region surrounding the scalarising neutron star (for example, it neglects the dynamical space-time curvature, the magneto-hydrodynamics of the collapsing baryons, and the finite size and internal structure of the remnant). Nevertheless, the scalar profiles plotted in Fig. 4 bear a close resemblance to the results of the full numerical simulation reported in Fig. 1 of [45] as well as the profiles for the self-interacting scalar fields in Figs. 1 and 2. The fact that the qualitative features of the true signal can be recovered by a simple toy model based solely on the massive wave equation (16) serves to emphasise the point that, for non-self-interacting scalar fields, the core collapse GW signal observed at large distances is determined almost entirely by the dispersive nature of the wave propagation in flat space-time, and not by the internal dynamics of the GW source. 
We now turn our attention to self-interacting scalar fields [i.e. $n>0$ in Eq. (7)]. The above analysis of our toy model cannot be repeated for this case because the flat space wave equation is now non-linear and does not admit a solution using Green's functions;

$$
\left(\partial_{t}^{2}-\nabla^{2}\right) \varphi+\mu^{2} \varphi\left(1+\lambda_{1} \varphi^{2}+\lambda_{2} \varphi^{4}+\ldots+\lambda_{n} \varphi^{2 n}\right)=4 \pi \varrho .
$$

However, the non-linear terms in this equation are only significant near the origin where $\lambda_{k} \varphi^{2 k} \sim 1$; at large distances the scalar field decays as $\varphi \lesssim 1 / r$ (or faster). Therefore, the propagation of the signal from the origin to some intermediate radius $r_{\mathrm{ex}}$ where $\lambda_{1} \varphi^{2} \ll 1$ is governed by the full non-linear equation. However, the subsequent propagation from $r_{\mathrm{ex}}$ out to the astrophysical distances $d$ relevant for LIGO/Virgo observations is once again governed, to an excellent approximation, by the linear Eq. (14). Therefore, we expect that at very large distances the signal will still be an inverse chirp as described by Eqs. (12) and (13).

\subsection{Numerical integration of the non-linear Klein-Gordon equation}

The expectation from the above discussion is that higher-order effects in the potential should not significantly affect the propagation of the gravitational wave signal provided a sufficiently large $r_{\mathrm{ex}}$ is chosen. We test this hypothesis by numerically solving the three dimensional Klein-Gordon equation (19) with $\varrho=0$; here we do not source a signal through some model function $\varrho$, but instead inject a genuine core collapse waveform at $r=r_{\mathrm{ex}}$ and propagate it further outwards to $r=d$ with the homogeneous Klein-Gordon equation. Writing this equation in terms of the variable $\sigma=r \varphi$ (and restoring factors of $\hbar$ ), we obtain a one-dimensional wave equation that we evolve in characteristic coordinates $(u:=t-r, r)$ in the form of the first-order system

$$
\begin{aligned}
& \partial_{r} \sigma=\eta, \quad \varphi=\frac{\sigma}{r}, \\
& \partial_{u} \eta=\frac{1}{2} \partial_{r} \eta-\frac{1}{2} \sigma \frac{\mu^{2}}{\hbar^{2}}\left(1+\lambda_{1} \varphi^{2}+\lambda_{2} \varphi^{4}+\ldots+\lambda_{n} \varphi^{2 n}\right) .
\end{aligned}
$$

The specific injected signal in our case is given by the waveform $\sigma\left(t, r_{\mathrm{ex}}\right)$ extracted at $r_{\mathrm{ex}}=7 \times 10^{4} \mathrm{~km}$ from the collapse of a $12 M_{\odot}$ progenitor in non-self-interacting ST gravity with $\alpha_{0}=0.01, \beta_{0}=-17$. This is a representative example of a waveform generated for a strongly scalarising star, but it here only serves as a common starting signal for the Klein-Gordon evolutions; what we are interested in is how the propagation of this signal from $r_{\mathrm{ex}}$ to $d$ changes for different choices of the $\lambda_{i}$ while setting all other $\lambda_{i}=0$ and fixing $\mu=10^{-14} \mathrm{eV}$.

The signal propagated to $d=3 \times 10^{7} \mathrm{~km}$ with $\lambda_{1}=0$ and $\lambda=10^{10}$, respectively, is shown by the two curves in the top row of Fig. 5. For $\lambda_{1}=10^{6}$ and $\lambda_{1}=10^{8}$ the curves would be indistinguishable from the $\lambda_{1}=0$ case in this plot and we therefore do not include them. The differences in the propagated signals for all self-interacting cases relative to the $\lambda_{1}=0$ signal are shown in the three remaining rows of Fig. 5; note that this deviation increases linearly with $\lambda_{1}$ but remains well below the percent level even for $\lambda_{1}=10^{8}$.

Even within the relatively simple framework of the characteristic Klein-Gordon equation, it is not feasible to compute the propagation of the signal to distances of the order of $10 \mathrm{kpc}$, typical for galactic LIGO sources. We have, however, repeated the above numerical analysis for $d=6 \times 10^{7} \mathrm{~km}$ to assess how our findings change 


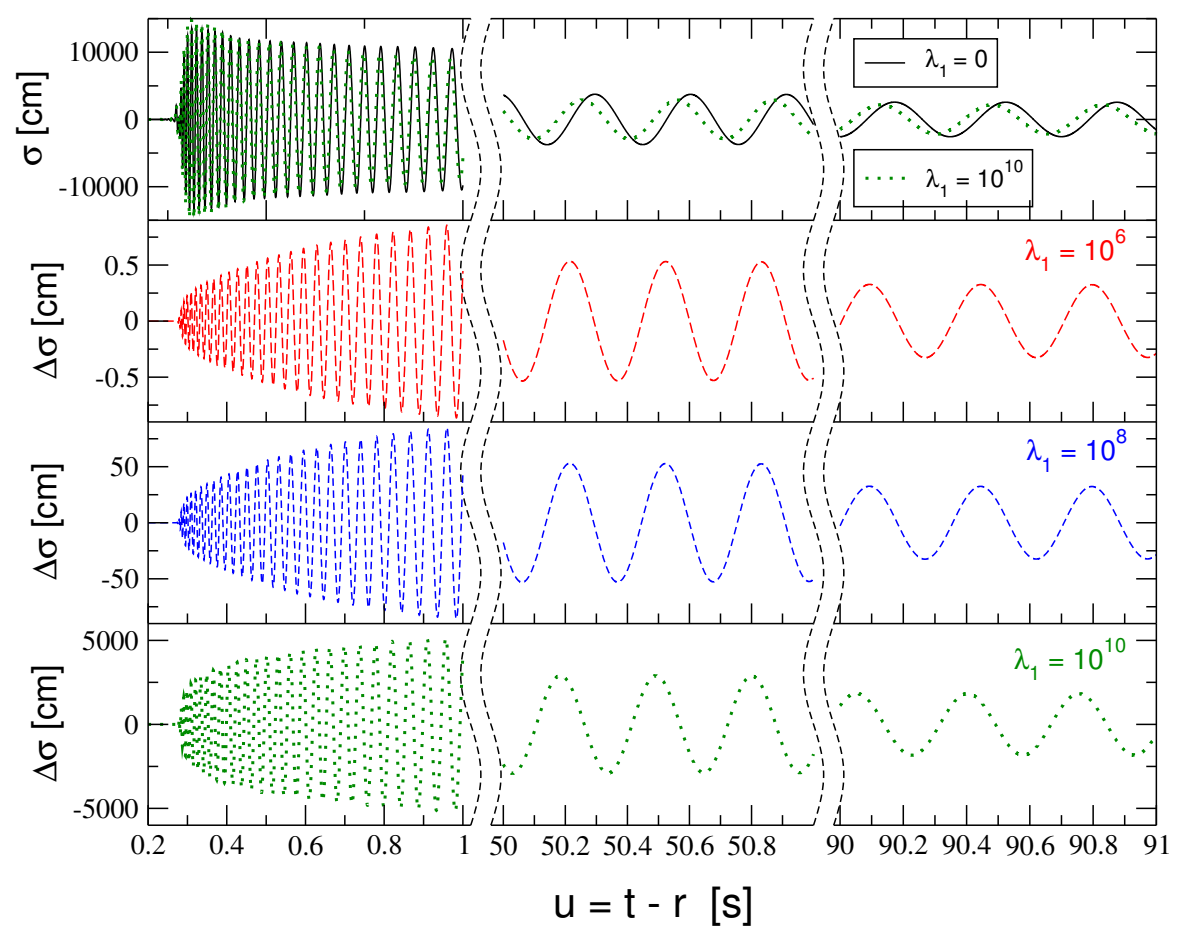

Figure 5. A representative scalar wave signal is injected at $r_{\mathrm{ex}}=7 \times 10^{4} \mathrm{~km}$ and then propagated according to Eq. (20) to $d=3 \times 10^{7} \mathrm{~km}$ for $\lambda_{1}=0,10^{6}, 10^{8}$ and $10^{10}$, respectively. The top row shows the signals thus obtained at $d=3 \times 10^{7} \mathrm{~km}$; the waveforms for $\lambda_{1}=10^{6}$ and $\lambda_{1}=10^{8}$ would be indistinguishable from the $\lambda_{1}=0$ curve and are not included to avoid confusion. Note that it would be impossible to resolve the long and highly oscillatory signal over its entire duration in this figure and we instead display 3 segments of length $1 \mathrm{~s}$ each around $\mathrm{u}=0,50$ and $90 \mathrm{~s}$. The three following rows respectively show the difference of the three signals propagated with self-interacting potential from the $\lambda_{1}=0$ case. Note that the deviation grows linearly with $\lambda_{1}$ and is negligible up to about $\lambda_{1}=10^{8}$.

with extraction radius. We find no significant difference; the analogous plot for $d=6 \times 10^{7} \mathrm{~km}$ is barely distinguishable from Fig. 5. In conclusion, the propagation of the wave signal in ST theory with higher-order self-interaction terms remains essentially unchanged relative to the massive case studied in Ref. [45], provided $\lambda_{1} \lesssim 10^{8}$. For larger values of $\lambda_{1}$, significant quantitative deviations arise but do not change the "inverse-chirp" character nor the oscillatory pattern of the signal. This result is in agreement with a rough estimate of the accumulation of deviations in the self-interacting case: Over a distance $d$, the leading extra terms in the potential are of order $\mathcal{O}\left(d^{-2}\right)$ and the total accumulated deviation is dominated by the contributions at small distance from the source. From Figs. 1-3, we see that the scalar field reaches values of the order of $10^{-5}$ at $r_{\mathrm{ex}}=7 \times 10^{4} \mathrm{~km}$, so that $\lambda_{1} \varphi^{2} \sim 1$ for $\lambda_{1}=10^{10}$, which is precisely the magnitude of $\lambda_{1}$ where deviations in the scalar wave propagation become significant.

For the sixth-order and higher terms in the potential, the same argument holds even more emphatically: The $\lambda_{2}$ term in the potential falls of with $d^{-4}$ and has even less impact on the wave propagation than the quartic terms and likewise for $\lambda_{3}$ etc. 


\section{Conclusions}

Scalar-tensor theories are perhaps the theoretically most well understood of all possible modifications to the general theory of relativity. In such theories compact objects, such as neutron stars, can undergo spontaneous scalarisation [18], where the scalar field inside the star grows rapidly to large values of order $\mathcal{O}(1)$. This process, and the accompanying GW signal produced, has been studied dynamically during the core collapse process by a number of authors [19, 20, 21, 22, 23, 24, 25, 26].

If the scalar is endowed with a mass (i.e. a quadratic potential) a much wider range of the theory's parameter space is compatible with present observational constraint, allowing for a significantly enhanced magnitude of the scalarisation, and the GW signal gets distorted by the effects of dispersion and tends to a universal inverse-chirp profile [45].

In this article we have investigated the effects of self-interaction in the scalar field (i.e. a more complicated potential including several higher-order terms). Our results should be compared with those of [46] where the case of the quartic potential was studied and it was found that self-interaction can suppress the scalarisation effect. Our results are consistent with this conclusion, but we note that such suppression requires a great deal of fine-tuning of the parameters of the potential. For example, when including a quartic $\varphi^{4}$ term, the dimensionless coefficient must be artificially set to a factor of $\gtrsim 10^{5}$ larger than the leading quadratic term before there is any noticeable effect (see Fig. 1). The main conclusion of our study is that the scalarisation phenomenon is incredibly robust against the effects of scalar self-interaction.

We have also demonstrated that the important features of the wave propagation are unaffected by the presence of self-interaction in the scalar potential. In particular, the observed signal still tends to the same universal inverse-chirp profile at large distances.

This apparent insensitivity to the detailed shape of the potential may be both a blessing and a curse. On the one hand it allows a very simple and robust prediction of the expected GW signal to be made. However, if such a signal should ever be detected it will make it very difficult to observationally study any details of physics in the scalar sector, beyond measuring the scalar field mass.

\section{Acknowledgments}

We thank Davide Gerosa, David Hilditch, and Christian Ott for helpful discussions. This work was supported by the European Union's H2020 ERC Consolidator Grant "Matter and strong-field gravity: New frontiers in Einstein's theory" grant agreement no. MaGRaTh-646597 funding from the European Union's Horizon 2020 research and innovation programme under the Marie Skłodowska-Curie grant agreement No 690904, the COST Action Grant No. CA16104, from STFC Consolidator Grant No. ST/P000673/1, the SDSC Comet and TACC Stampede2 clusters through NSFXSEDE Award Nos. PHY-090003, and Cambridge's CSD3 system system through STFC capital grants ST/P002307/1 and ST/R002452/1 and STFC operations grant ST/R00689X/1. R.R.-M. acknowledges support by a STFC studentship. 


\section{References}

[1] D. Psaltis. Probes and Tests of Strong-Field Gravity with Observations in the Electromagnetic Spectrum. Living Rev. Rel., 11:9, 2008.

[2] Clifford M. Will. The Confrontation between General Relativity and Experiment. Living Rev. Rel., 17:4, 2014.

[3] Emanuele Berti et al. Testing General Relativity with Present and Future Astrophysical Observations. Class. Quant. Grav., 32:243001, 2015. arXiv:1501.07274 [gr-qc].

[4] B. P. Abbott et al. Observation of Gravitational Waves from a Binary Black Hole Merger. Phys. Rev. Lett., 116(6):061102, 2016. arXiv:1602.03837 [gr-qc].

[5] GWTC-1: A Gravitational-Wave Transient Catalog of Compact Binary Mergers Observed by LIGO and Virgo during the First and Second Observing Runs. 2018. arXiv:1811.12907 [astro-ph].

[6] J. Aasi et al. Advanced LIGO. Class. Quant. Grav., 32:074001, 2015.

[7] F. Acernese et al. Advanced Virgo: a second-generation interferometric gravitational wave detector. Class. Quant. Grav., 32(2):024001, 2015.

[8] Tests of General Relativity with the Binary Black Hole Signals from the LIGO-Virgo Catalog GWTC-1. 2019. arXiv:1903.04467 [gr-qc].

[9] Ny Yunes, K. Yagi, and F. Pretorius. Theoretical Physics Implications of the Binary Black-Hole Mergers GW150914 and GW151226. Phys. Rev., D94(8):084002, 2016.

[10] B. P. Abbott et al. Tests of general relativity with GW150914. Phys. Rev. Lett., 116(22):221101, 2016. arXiv:1602.03841 [gr-qc].

[11] B. P. Abbott et al. Tests of General Relativity with GW170817. 2018.

[12] T. Delsate, D. Hilditch, and H. Witek. The Initial Value Formulation of Dynamical ChernSimons Gravity. Phys. Rev. D, 91:024027, 2014. arXiv:1407.6727 [gr-qc].

[13] Giuseppe Papallo and Harvey S. Reall. On the local well-posedness of Lovelock and Horndeski theories. Phys. Rev. D, 96(4):044019, 2017.

[14] Gwyneth Allwright and Luis Lehner. Towards the nonlinear regime in extensions to GR: assessing possible options. 2018.

[15] G. Papallo. Causality and the initial value problem in Modified Gravity. PhD thesis, Cambridge U., DAMTP, 2018-03-29.

[16] M. Okounkova, L. C. Stein, M. A. Scheel, and D. A. Hemberger. Numerical binary black hole mergers in dynamical Chern-Simons gravity: Scalar field. Phys. Rev. D, 96(4):044020, 2017.

[17] H. Witek, L. Gualtieri, P. Pani, and T. P. Sotiriou. Black holes and binary mergers in scalar Gauss-Bonnet gravity: scalar field dynamics. 2018.

[18] T. Damour and G. Esposito-Farèse. Nonperturbative strong field effects in tensor - scalar theories of gravitation. Phys. Rev. Lett., 70:2220-2223, 1993.

[19] M. A. Scheel, S. L. Shapiro, and S. A. Teukolsky. Collapse to black holes in Brans-Dicke theory. I. Horizon boundary conditions for dynamical systems. Phys. Rev. D, 51:4208-4235, 1995.

[20] T. Harada, T. Chiba, K.-i. Nakao, and T. Nakamura. Scalar gravitational wave from Oppenheimer-Snyder collapse in scalar - tensor theories of gravity. Phys. Rev. D, 55:20242037, 1997.

[21] J. Novak. Spherical neutron star collapse toward a black hole in a tensor-scalar theory of gravity. Phys. Rev. D, 57:4789-4801, 1998. gr-qc/9707041.

[22] J. Novak. Neutron star transition to strong scalar field state in tensor scalar gravity. Phys. Rev. D, 58:064019, 1998. gr-qc/9806022.

[23] J. Novak and J. M. Ibáñez. Gravitational waves from the collapse and bounce of a stellar core in tensor scalar gravity. Astrophys. J., 533:392-405, 2000. astro-ph/9911298.

[24] C. Palenzuela, E. Barausse, M. Ponce, and L. Lehner. Dynamical scalarization of neutron stars in scalar-tensor gravity theories. Phys. Rev. D, 89:044024, 2014. arXiv:1310.4481 [gr-qc].

[25] C. Palenzuela and S. L. Liebling. Constraining scalar-tensor theories of gravity from the most massive neutron stars. Phys. Rev. D, 93(4):044009, 2016.

[26] D. Gerosa, U. Sperhake, and C. D. Ott. Numerical simulations of stellar collapse in scalar-tensor theories of gravity. Class. Quant. Grav., 33(13):135002, 2016. arXiv:1602.06952 [gr-qc].

[27] Laura Bernard. Dynamics of compact binary systems in scalar-tensor theories: Equations of motion to the third post-Newtonian order. Phys. Rev., D98(4):044004, 2018.

[28] Laura Bernard. Dynamics of compact binary systems in scalar-tensor theories: II. Center-ofmass and conserved quantities to 3PN order. Phys. Rev., D99(4):044047, 2019.

[29] P. C. C. Freire et al. The relativistic pulsar-white dwarf binary PSR J1738+0333 II. The most stringent test of scalar-tensor gravity. Mon. Not. Roy. Astron. Soc., 423:3328, 2012. arXiv:1205.1450 [astro-ph]. 
[30] J. Antoniadis et al. A Massive Pulsar in a Compact Relativistic Binary. Science, 340:1233232, 2013. arXiv:1304.6875 [qstro-ph].

[31] Norbert Wex. Testing Relativistic Gravity with Radio Pulsars. 2014.

[32] B. Bertotti, L. Iess, and P. Tortora. A test of general relativity using radio links with the Cassini spacecraft. Nature, 425:374-376, 2003.

[33] J. Alsing, E. Berti, C. M. Will, and H. Zaglauer. Gravitational radiation from compact binary systems in the massive Brans-Dicke theory of gravity. Phys. Rev. D, 85:064041, 2012.

[34] F. M. Ramazanoglu and F. Pretorius. Spontaneous Scalarization with Massive Fields. Phys. Rev. D, 93(6):064005, 2016. arXiv:1601.07475 [gr-qc].

[35] T. Damour and G. Esposito-Farése. Tensor multiscalar theories of gravitation. Class. Quant. Grav., 9:2093-2176, 1992.

[36] T. Damour and G. Esposito-Farése. Tensor - scalar gravity and binary pulsar experiments. Phys. Rev. D, 54:1474-1491, 1996. gr-qc/9602056.

[37] M. Salgado. The cauchy problem of scalar-tensor theories of gravity. Class. Quantum Grav., 23:4719-4741, 2006. gr-qc/0509001.

[38] M. Salgado, D. Martínez-del Río, M. Alcubierre, and D. Nuñez. Hyperbolicity of scalar-tensor theories of gravity. Phys. Rev. D, 77:104010, 2008. arXiv:0801.2372 [gr-qc].

[39] D. D. Doneva and S. S. Yazadjiev. Rapidly rotating neutron stars with a massive scalar field - structure and universal relations. JCAP, 1611(11):019, 2016.

[40] S. S. Yazadjiev, D. D. Doneva, and D. Popchev. Slowly rotating neutron stars in scalar-tensor theories with a massive scalar field. Phys. Rev. D, 93(8):084038, 2016.

[41] D. D. Doneva, S. S. Yazadjiev, N. Stergioulas, and K. D. Kokkotas. Differentially rotating neutron stars in scalar-tensor theories of gravity. Phys. Rev. D, 98(10):104039, 2018.

[42] M. Horbatsch, H. O. Silva, D. Gerosa, P. Pani, E. Berti, L. Gualtieri, and U. Sperhake. Tensor-multi-scalar theories: relativistic stars and 3+1 decomposition. Class. Quant. Grav., 32(20):204001, 2015. arXiv:1505.07462 [gr-qc].

[43] F. M. Ramazanoğlu. Spontaneous growth of vector fields in gravity. Phys. Rev. D, 96(6):064009, 2017.

[44] F. M. Ramazanoğlu. Spontaneous tensorization from curvature coupling and beyond. 2019.

[45] U. Sperhake, C. J. Moore, R. Rosca, M. Agathos, D. Gerosa, and C. D. Ott. Long-lived inverse chirp signals from core collapse in massive scalar-tensor gravity. Phys. Rev. Lett., 119(20):201103, 2017.

[46] P. C.-K. Cheong and T. G. F. Li. Numerical Studies on Core Collapse Supernova in Selfinteracting Massive Scalar-Tensor Gravity. 2018.

[47] P. G. Bergmann. Comments on the scalar tensor theory. Int. J. Theor. Phys., 1:25-36, 1968.

[48] R. V. Wagoner. Scalar tensor theory and gravitational waves. Phys. Rev. D, 1:3209-3216, 1970.

[49] Y. Fujii and K. Maeda. The scalar-tensor theory of gravitation. Cambridge University Press, 2007.

[50] S. L. Shapiro and S. A. Teukolsky. Black Holes, White Dwarfs, and Neutron Stars. John Wiley \& Sons, Inc., 1983.

[51] Takeshi Chiba, Tomohiro Harada, and Ken-ichi Nakao. Gravitational physics in scalar tensor theories: Tests of strong field gravity. Prog. Theor. Phys. Suppl., 128:335-372, 1997.

[52] E. O'Connor and C. D. Ott. A New Open-Source Code for Spherically-Symmetric Stellar Collapse to Neutron Stars and Black Holes. Class.Quant.Grav., 27:114103, 2010. arXiv:0912.2393 [astro-ph].

[53] S. E. Woosley and A. Heger. Nucleosynthesis and Remnants in Massive Stars of Solar Metallicity. Phys. Rept., 442:269-283, 2007. astro-ph/0702176.

[54] https://2sn.org/stellarevolution/.

[55] S. W. Hawking. Black holes in the Brans-Dicke Theory of gravitation. Comm. Math. Phys., 25:167-171, 1972.

[56] K. S. Thorne and J. J. Dykla. Black Holes in the Dicke-Brans Theory of Gravity. Astrophys. J. Lett., 166:L35, 1971.

[57] U. Sperhake, C. J. Moore, R. Rosca, M. Agathos, D. Gerosa, and C. D. Ott. Follow up to the earlier letter, in prep.

[58] E. Poisson. The Motion of Point Particles in Curved Spacetime. Living Reviews in Relativity, 7(6), 2004. http://www.livingreviews.org/lrr-2004-6. 